\title{
Electronic neuron within a ganglion of a leech (Hirudo medicinalis)
}

\author{
J. Aliaga, ${ }^{1}$ N. Busca, ${ }^{1}$ V. Minces, ${ }^{1}$ G. B. Mindlin, ${ }^{1}$ B. Pando, ${ }^{1}$ A. Salles, ${ }^{1}$ and L. Sczcupak ${ }^{2}$ \\ ${ }^{1}$ Laboratorio de Sistemas Dinámicos, Departamento de Física, FCEN, UBA, Ciudad Universitaria, Pabellón I (1428), \\ Buenos Aires, Argentina \\ ${ }^{2}$ Departamento de Fisiología, Biología Molecular y Celular, FCEN, UBA Ciudad Universitaria, Pabellón II (1428), \\ Buenos Aires, Argentina
}

(Received 30 December 2002; revised manuscript received 2 April 2003; published 27 June 2003)

\begin{abstract}
We report the construction of an electronic device that models and replaces a neuron in a midbody ganglion of the leech Hirudo medicinalis. In order to test the behavior of our device, we used a well-characterized synaptic interaction between the mechanosensory, sensitive to pressure, $(\mathrm{P})$ cell and the anteropagoda (because of the action potential shape) (AP) neuron. We alternatively stimulated a P neuron and our device connected to the AP neuron, and studied the response of the latter. The number and timing of the AP spikes were the same when the electronic parameters were properly adjusted. Moreover, after changes in the depolarization of the AP cell, the responses under the stimulation of both the biological neuron and the electronic device vary in a similar manner.
\end{abstract}

DOI: 10.1103/PhysRevE.67.061915

PACS number(s): 87.17.Nn, 87.80.Tq

\section{INTRODUCTION}

In the past years, there has been a growing interest in the physics community for biologically inspired problems. From the development of new measuring devices to the dynamics of living units, a wide range of techniques and models have been explored. Among these problems, the individual and collective behaviors of neurons in nervous systems have got considerable attention. Within this line of research, at every scale of the question interesting phenomena arise, from the dynamics of ion channels to the computational capability of a set of interconnected neurons.

The modeling of the dynamics of neurons offered a wide range of possibilities. The simplest conceivable model is probably the one used in the neural network community, where neurons are a single compartment that adds incoming signals until a threshold is achieved. The introduction of the dynamics of ionic conductances and multiple processing compartments can make a model virtually as complex as desired. Which approach might be convenient will depend on the question being addressed. The success of a modeling effort is, of course, given by experimental validation, i.e., the possibility of emulating an experimental observation or performing predictions.

In the past years, an even more challenging validation scheme has been proposed: the construction of a physical device that analogically integrates the functions of a given neuron according to a specific model. This device is, then, used to replace the neuron and test its functioning within the natural neuronal network. In this strategy the validation of the model results from the quantification of the similarities between the performance of the neuronal network in the natural configuration, and when one of its neurons was replaced by the analogical device [1].

In this work, we report the construction of an electronic device that models and replaces a neuron in a midbody ganglion of the leech Hirudo medicinalis. The presentation of this work is organized as follows. In Sec. II, we review previous efforts in the construction of electronic neurons. In
Sec. III, we describe the model ruling the dynamics of our electronic device and its description is included in Sec. IV. The biological experiences are reported in Sec. V. Discussion and conclusions are presented in Sec. VI.

\section{ELECTRONIC NEURONS}

Excitability is the most interesting dynamical feature. A system is said to be excitable according to its response to an external stimulus. An excitable system will (in the absence of noise) stay in its resting position unless it is perturbed. If the perturbation is smaller than a given threshold, the system will decay almost linearly to its equilibrium position. The behavior will be different if the perturbation exceeds the threshold: in this case, the system will explore a large region of its phase space before returning to the equilibrium state. This dynamical feature offers a rich variety of behaviors under time dependent perturbations. If these are periodic, different kinds of locking states can be achieved. If the system is forced with an arbitrary time dependent signal, its response will be extremely complex. As an example of an early study of forced excitable systems, Feingold et al. reported an extensive research in which such forced systems displayed phase locking, period doubling, and chaos. Even if the work consisted of numerical simulations of a simple set of equations, the authors described a possible electronic realization which could qualitatively display the features under study [2].

Actually, the interest in excitability lies mainly in the fact that this feature is present in the behavior of neurons, in a phenomenon known as action potentials [3]. The original work of Hodgkin and Huxley provided us with a phenomenological model of these action potentials. This model is rather complex, since it involves a prescription for the dynamics of the ionic conductances underlying the voltage changes. A simplified model, widely used in the literature, was proposed by FitzHugh [4] and Nagumo [5] (FN).

This FN system served as a paradigm for excitability (more than other models displaying excitability) for its strong biological inspiration [6]. One of the aspects explored using this model was the behavior of (eventually forced) ex- 
citable systems under noise. For example, stochastic resonance (the noise-induced amplification of signals by some nonlinear devices) was tested in this model (among others). Before testing the existence of this phenomenon in biological excitable neurons, Moss et al. implemented an electronic device whose behavior is described by the FN equations [7].

More recently, electronic neurons were built by Szucs et al. [1]. The remarkable aspect of this work is that this device was connected by means of an artificial tunable synapse to biological neurons of the central pattern generator of the stomatogastric ganglion of a spiny lobster. The electronic neuron was built in such a way that its behavior would be ruled by the equations of the Hindmarsh and Rose model [8]. These equations are able to display chaotic behavior, and yet the electronic neuron is able to produce regular rhythms when coupled with the biological neural circuit. But most important, their results suggest that it is not necessary to reproduce all of the biological aspects of the cells to reproduce some relevant emergent collective behavior.

From a dynamical point of view, it is difficult to address the problem of classifying the qualitatively different types of excitable systems. The core of this difficulty lies in the fact that excitability is a global property and, therefore, the normal form theory is of little help. Yet, the simplest excitable models (describable in terms of bidimensional vector fields) can be classified (following Hodgkin and Huxley) as classes I and II [9]. In class-I systems, the transition from excitability to periodic spiking is mediated by a saddle node homoclinic bifurcation (also known as a Andronov bifurcation), while in class-II systems this transition takes place through a (degenerate) Andronov-Hopf bifurcation [10]. This difference is not minor: the oscillations born in saddle node homoclinic bifurcations are of zero frequency, while the ones being born in Andronov-Hopf bifurcations are characterized by frequencies of finite value. For this reason, weakly coupled systems of one or the other behave differently [9]. Moreover, many biological circuits mix both types of excitabilities in a given architecture [11].

Modeling of a neuron within a network requires one to emulate the excitable properties of the neuron and also the mechanism by which it communicates with the other neurons, the synapse. Different models for synapses can be stated according to the kind of neurotransmitters and receptors involved [12]. Given the multiple variables in the inputoutput processing of any given neuron, it is pertinent to work out the program for analyzing the degree of adequate simplification in the modeling of neural behavior. Replacement of neurons by electronic devices, ruled by the equations of simple models, can contribute to the understanding of critical biological variables at play.

\section{MODEL AND DEVICE}

In this work, we built an excitable device capable of generating the dynamical responses of class-I excitable systems. As a model representative of these systems, we write

$$
V^{\prime}=W
$$

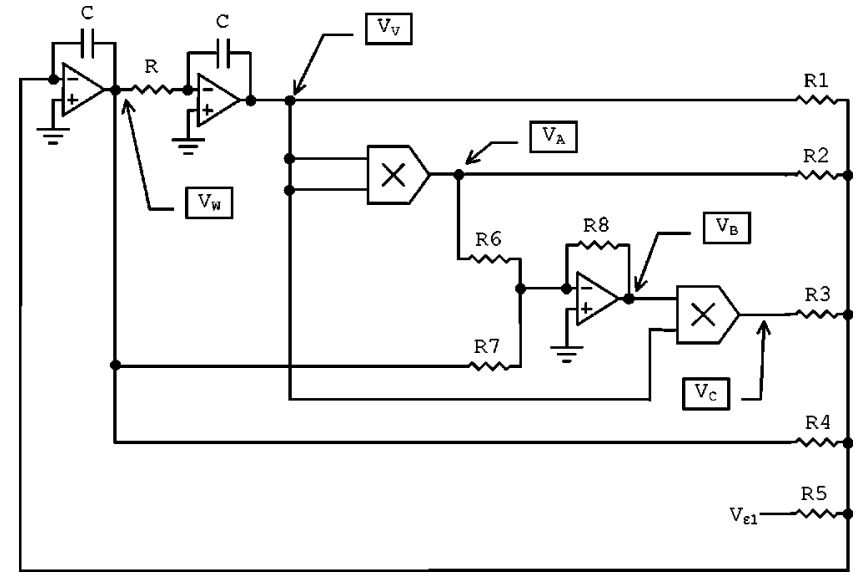

FIG. 1. Electronic neuron. The output of the circuit is $V_{v}$. The multiplication is obtained using AD633. $R=10 \mathrm{k} \Omega, R_{1}=68 \mathrm{k} \Omega$, $R_{2}=25 \mathrm{k} \Omega, R_{3}=1 \mathrm{k} \Omega, R_{4}=27 \mathrm{k} \Omega, R_{5}=100 \mathrm{k} \Omega, R_{6}=2.2 \mathrm{k} \Omega$, $R_{7}=4.7 \mathrm{k} \Omega, R_{8}=1 \mathrm{k} \Omega$, and $C=0.1 \mu \mathrm{F}$.

$$
W^{\prime}=V-W-V^{3}+V W+\epsilon_{1}+\epsilon_{2} V^{2}
$$

where $V$ stands for the voltage across a membrane, and $\epsilon_{1}$ and $\epsilon_{2}$ are real parameters. This system of equations was widely studied in the literature $[10,13,14]$. For appropriate values of the constants $\epsilon_{1}$ and $\epsilon_{2}$, the system behaves as an excitable one. As $\epsilon_{1}$ is increased, the system loses its excitability through an Andronov bifurcation that leads to periodic oscillations. An advantage of this system as a model for excitable neuron dynamics is that both dynamical variables $V$ and $W$ can be measured from the voltage recordings (the first one is just the recorded variable, while the second can be computed from the time series). In this way, it is simple to fit the values of the constants from the easiest recordable variable of the problem. Scaling the variables $V \rightarrow \alpha V, t \rightarrow \tau t$, and $W \rightarrow(\alpha / \tau) W$, it is possible to fit the duration of the pulses generated with these equations to the duration of the depolarizing pulses in neurons. The equations after performing the scaling read

$$
\begin{gathered}
V^{\prime}=W \\
W^{\prime}=\tau^{2} V-\tau W-\alpha^{2} \tau^{2} V^{3}+\alpha \tau V W+\frac{\tau^{2}}{\alpha} \epsilon_{1}+\alpha \tau^{2} \epsilon_{2} V^{2}
\end{gathered}
$$

With $\tau=0.38$, the integration time in milliseconds allows us to reproduce the observed duration of the spikes displayed by the neurons of our biological preparations. The scaling $\alpha$ was fitted to bound the voltages within the saturation limits of our devices. Since the output of our neuron was connected to an electronic synapse, it was not important to obtain the same values of voltages as those in the biological neurons. A circuit ruled by these equations is displayed in Fig. 1. In this figure, the voltages at different points are indicated by an inset. These are $V_{w}, V_{v}, V_{a}, V_{b}$, and $V_{c}$. Following the circuit clockwise from the top, we see that $V_{v}=$ $-(1 / R C) \int V_{w} d t$. The voltage $V_{a}$ is obtained by an AD633 multiplier and reads $V_{a}=V_{v}^{2} /(10$ volt $)$. The voltage $V_{b}$ is the 
result of adding the fractions of $V_{a}$ and $V_{w}$ with a summing amplifier. A multiplier is used to obtain $V_{c}$ $=V_{b} V_{v} /(10$ volt $)$. Finally, an integrator generates $V_{w}$ integrating a sum of terms that are proportional to the voltages described above, added to a constant voltage, i.e., $V_{w}=-(1 / C) \int\left[\left(V_{v} / R_{1}\right)+\left(V_{a} / R_{2}\right)+\left(V_{c} / R_{3}\right)+\left(V_{w} / R_{4}\right)\right.$ $\left.+\left(V_{\epsilon_{1}} / R_{5}\right)\right] d t$. In this way, the behavior of $V_{v}$ is ruled by

$$
\begin{gathered}
\frac{d V_{v}}{d t}=\frac{-V_{w}}{R C}+J_{\text {input }}, \\
\frac{d V_{w}}{d t}=\frac{-V_{v}}{R_{1} C}-\frac{V_{v}^{2}}{R_{2} C(10 \text { volts })}+\frac{R_{8} V_{v}^{3}}{R_{3} R_{6} C(10 \text { volts })^{2}} \\
+\frac{R_{8} V_{w} V_{u}}{R_{3} R_{7} C(10 \text { volts })}-\frac{V_{w}}{R_{4} C}-\frac{V_{\epsilon_{1}}}{R_{5} C},
\end{gathered}
$$

where $R_{i}(i=1, \ldots, 8)$ and $C$ stand for the resistances and capacitances displayed in Fig. 1. Defining $W=$ $-(1 / R C) V_{w}$, and choosing the values of resistances and capacitances listed in the caption of Fig. 1, these equations read as Eq. (2). The term $J_{\text {input }}$ represents a forcing input current.

After modeling the excitable nature of a neuron, our next step was to build an interface that would allow us to connect our device with a biological neuron. In order to do so, we model the synapse (following Ref. [15]) by a simple set of equations derived from the first-order kinetics that also incorporates the dynamics of neurotransmitter concentration $n$. Action potentials induce the release of neurotransmitters from the spiking cell in the synaptic space. The neurotramitters bind to ligand gated channels, increasing the conductivity of the postsynaptic neuron. A simple model accounting for these processes can be written as

$$
\frac{d n}{d t}=\Theta\left(V_{v, p r e}-V_{t h r}\right)-\gamma n
$$

and

$$
J=g_{0}\left(V_{v, p o s}-V_{r e v}\right)\left(\frac{1}{1+e^{-\lambda\left(n-n_{0}\right)}}-\frac{1}{1+e^{\lambda n_{0}}}\right),
$$

where $\Theta$ stands for the positive function (the source of neurotransmitter release is the existence of peaks) and $\gamma$ stands for the neurotransmitter loss rate. $V_{v \text {,pre }}$ and $V_{v, \text { pos }}$ are the presynaptic and postsynaptic voltages, $V_{t h r}$ is the voltage above which the presynaptic cell releases neurotransmitters, and $V_{r e v}$ is the potential at which the ionic current reverses its direction. The parameters $g_{0}, \lambda$, and $n_{0}$ characterize the saturation nature of the current: $g_{0}$ scales the conductivity, while $\lambda$ and $n_{0}$ determine the steepness and the midpoint of the saturation, respectively.

The circuit displayed in Fig. 2 implements the artificial synapse used to connect the excitable part of our device with a neuron. In Fig. 2(a), we show the part of the circuit in charge of performing the integration in Eq. (4). The first block implements the $\Theta$ function, while the second block performs the integration. The resistance $R_{9}$ controls the value
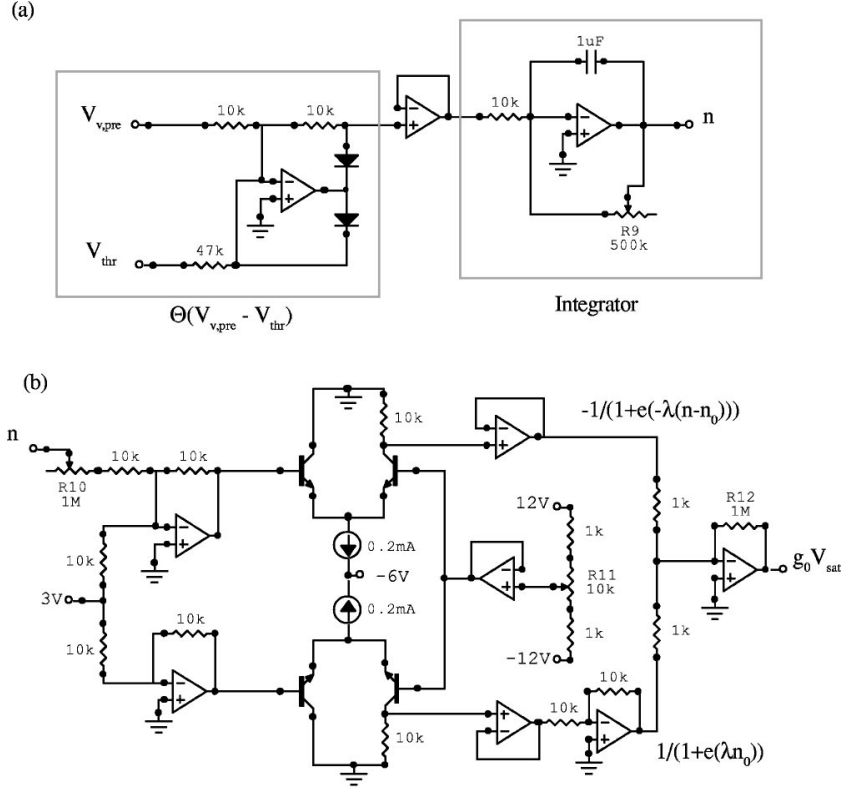

(c)

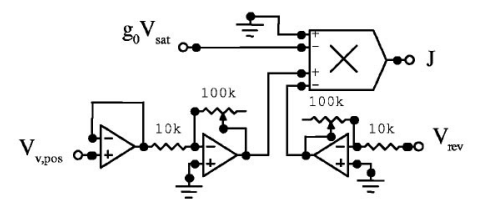

FIG. 2. Electronic synapse: (a) circuit in charge of performing the integration in Eq. (4). The first block implements the $\Theta$ function, while the second block performs the integration. (b) Circuit that produces a voltage $g_{0} v_{\text {sat }}$ [see Eq. (6)]. (c) This circuit evaluates the difference between postsynaptic potential at $V_{v, p o s t}$ and the constant $V_{\text {rev }}$ and multiplies it (using AD633 multiplier) with the output of the circuit shown in (b).

of $\gamma$, and the specific values of the resistances used in our implementation are given in the figure caption. The output of this circuit is a voltage that represents the value of $n$, and constitutes the input of the circuit in Fig. 2(b). This second circuit produces a voltage $g_{0} v_{\text {sat }}$ such that

$$
v_{s a t}=\frac{1}{1+e^{-\lambda\left(n-n_{0}\right)}}-\frac{1}{1+e^{\lambda n_{0}}},
$$

where $\lambda$ is controlled by the resistance $R_{10}$. The value of $\lambda n_{0}$ is in turn given by the potentiometer $R_{11}$. This circuit operates basically as two matched differential amplifiers. A current proportional to $v_{s a t}$ is multiplied by a constant $g_{0}$ that is controlled by the resistance $R_{12}$.

The last part of the artificial synapse is displayed in Fig. 2 (c), which senses the postsynaptic potential at $V_{v, p o s}$. The difference between this voltage and the constant $v_{\text {rev }}$ is multiplied with the output of the circuit shown in Fig. 2(b). Before discussing the results obtained when our device was connected to a neuron, we will discuss some tests on our circuits. 

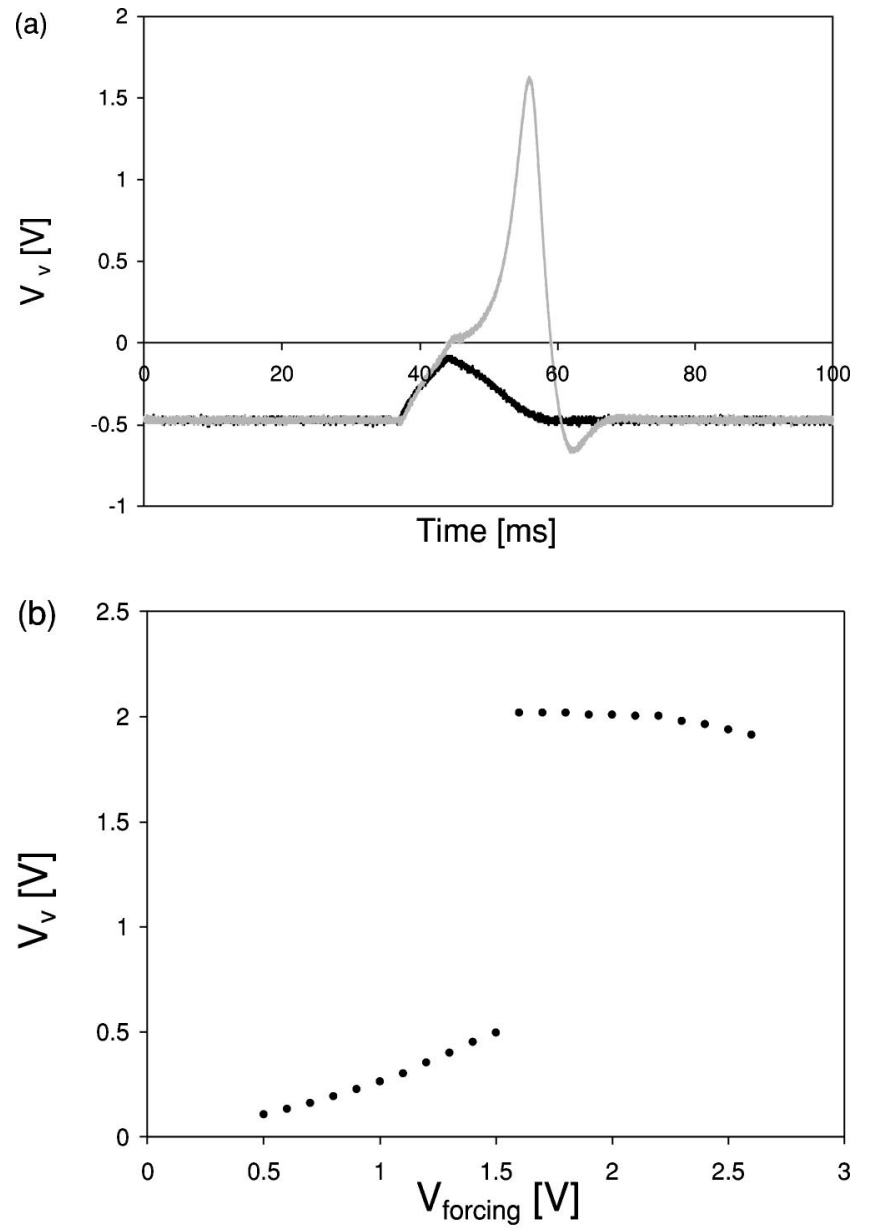

FIG. 3. Evidence of the excitable nature of the dynamical response of Eqs. (3) to external perturbations. The circuit representing Eqs. (3) was excited with an external forcing consisting of square impulses of different amplitudes lasting $7 \mathrm{~ms}$. (a) The dark line shows the typical response when the exciting amplitude $(1.2 \mathrm{~V})$ was below the threshold, while the gray line displays the global exploration of the values, which is obtained when the forcing amplitude exceeds the threshold $(1.4 \mathrm{~V})$. (b) $V_{v}$ for different values of the forcing amplitudes that are consistent with the type-I excitability $\left(R_{2}=18 \mathrm{k} \Omega\right)$.

\section{TEST}

In this section, we discuss some of the tests implemented in order to check the operational features of our electronic device. The first test consisted of a direct observation of the excitable nature of its dynamical response to external perturbations. In order to do so, we excited the circuit representing Eqs. (3) with an external forcing consisting of square pulses of different amplitudes lasting $7 \mathrm{~ms}$. In Fig. 3(a), with dark line we show the typical response when the exciting amplitude was below the threshold, while the gray line displays the global exploration of values that is obtained when the forcing amplitude exceeds the threshold. In Fig. 3(b), we show that, for different values of the forcing amplitudes, first a clear threshold exists and second, that once the threshold is exceeded, the amplitude of the response is basically independent of the forcing amplitude.

As we have discussed, for excitable regimes which can be
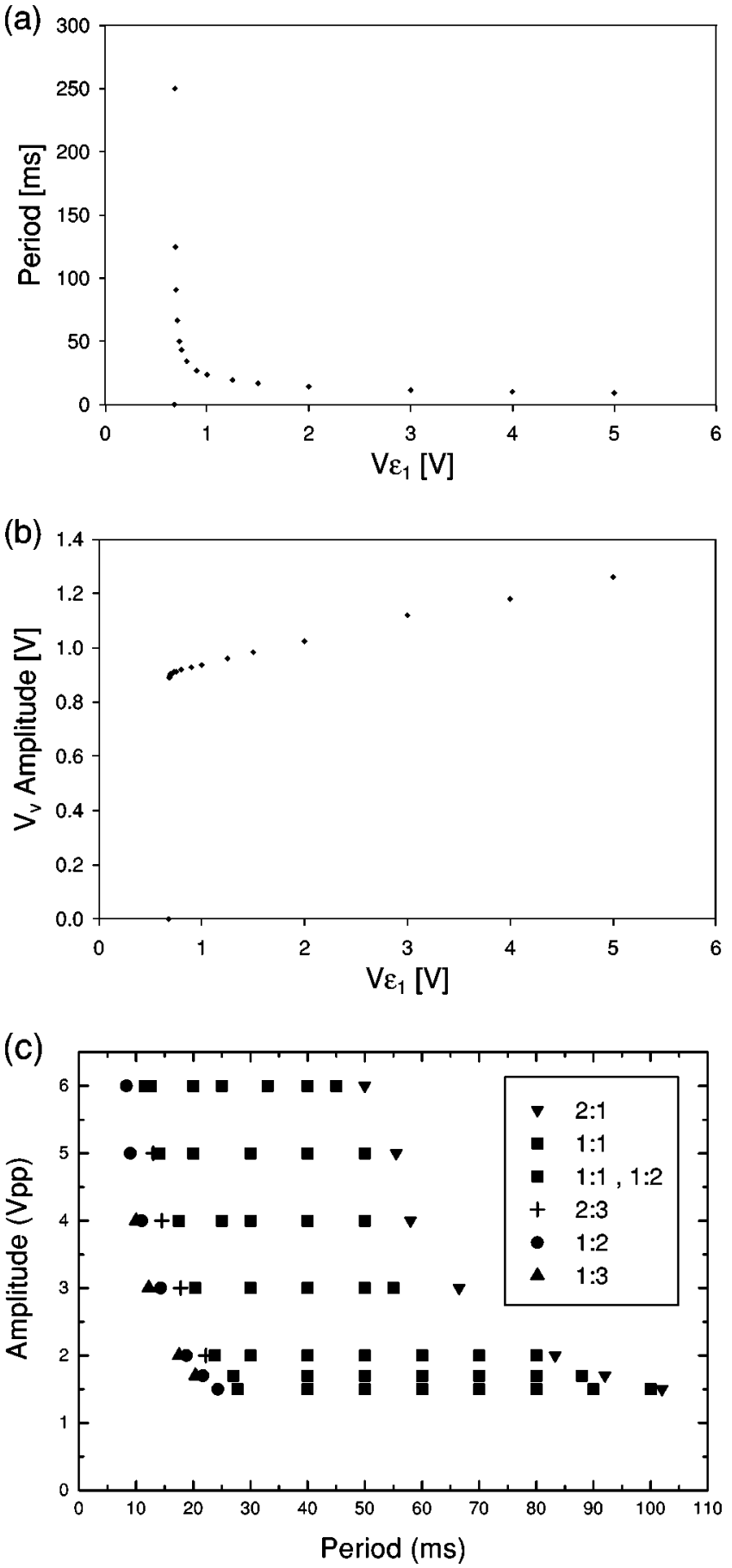

FIG. 4. (a) The periods of the oscillations obtained when no forcing is applied as a function of $V_{\epsilon_{1}}\left(R_{2}=15 \mathrm{k} \Omega\right)$. (b) The amplitude of the oscillations obtained when no forcing is applied as a function of $V_{\epsilon_{1}}\left(R_{2}=15 \mathrm{k} \Omega\right)$. (c) Rotation numbers $q$ : $p$ of periodic orbits observed as a function of the amplitude and frequency of the applied sinusoidal external forcing $\left(V_{\epsilon_{1}}=0.4 \mathrm{~V}, R_{2}=15 \mathrm{k} \Omega\right)$.

represented by two-dimensional dynamical systems, two different scenarios exist: type-I and type-II excitability. In the first class, the excitability is lost through a saddle node homoclinic bifurcation. This means that for constant excitatory inputs, the oscillations are born with an infinite period. To check that our circuit reproduces this feature, we show in 


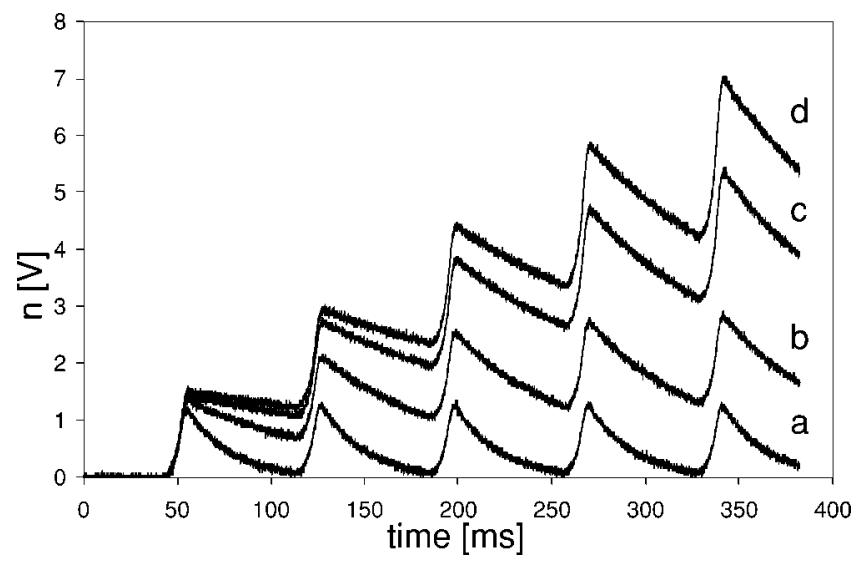

FIG. 5. Integration of $n$, the density of neurotransmitters, as a function of the the resistance $R_{9}$. (a) $R_{9}=15 \mathrm{k} \Omega$, (b) $R_{9}$ $=100 \mathrm{k} \Omega$, (c) $R_{9}=270 \mathrm{k} \Omega$, and (d) $R_{9}=430 \mathrm{k} \Omega$. We forced the excitable circuit with a train of five pulses of $7 \mathrm{~ms}$ separated by 66 ms.

Fig. 4(a) the periods of the auto-oscillations obtained when no forcing is applied as a function of the parameter $V_{\epsilon_{1}}$. Figure 4(b) shows the amplitude of the auto-oscillations as a function of the parameter $V_{\epsilon_{1}}$ when no forcing is applied.

We have also applied an electrical sinusoidal forcing into the circuit and have analyzed the response as the amplitude and the frequency of the forcing are changed. In this case, we have prepared the system far from the saddle-loop global bifurcation, as in the biological experiments, by choosing $V_{\epsilon_{1}}=0.4 \mathrm{~V}$ and $R_{2}=15 \mathrm{k} \Omega$. In Fig. 4(c), we show the experimental map of the regions in which the different periodical paterns with rotation number $q: p$ (i.e., the time series have $q$ spikes every $p$ periods of the forcing signal) have been observed $[2,16]$. The Arnold tongues bend towards higher periods as the forcing amplitude diminishes, as expected for an excitable system both of class I [16] or II [2].

The artificial synapse begins with the integration of $n$, representing the density of neurotransmitters. In order to inspect the effect of the parameters in Eq. (4) in this part of the processing, we forced the excitable circuit with a train of five pulses of $7 \mathrm{~ms}$ separated by $66 \mathrm{~ms}$. The behavior observed in the variable $n$ shows the effect of the integration controlled by the parameter $\gamma$. For small values of this constant, the reponses to the forcing inputs add up, as shown in Fig. 5. After accounting for the dynamics of the concentration of neurotransmitters $n$, the artificial synapse weights this concentration in a highly nonlinear way, represented by a sigmoidal function. This implies that the postsynaptic response will show no difference if the concentration of neurotransmitters is much higher than a given value. Our circuit implementing this nonlinear effect is ruled by Eq. (6). The outputs of this device for different values of $n$ is shown in Fig. 6. In these outputs, different values of $\lambda$ and $\lambda n_{0}$ are used. Notice that $\lambda$ controls the amplitude of the derivative of the sigmoidal response, and $\lambda n_{0}$ controls where the maximum growth of the slope takes place. The final results of this artificial synapse was tested taking a constant value of $V_{v, p o s}-V_{\text {ref }}$ and forcing the circuit representing Eqs. (3) with a set of five

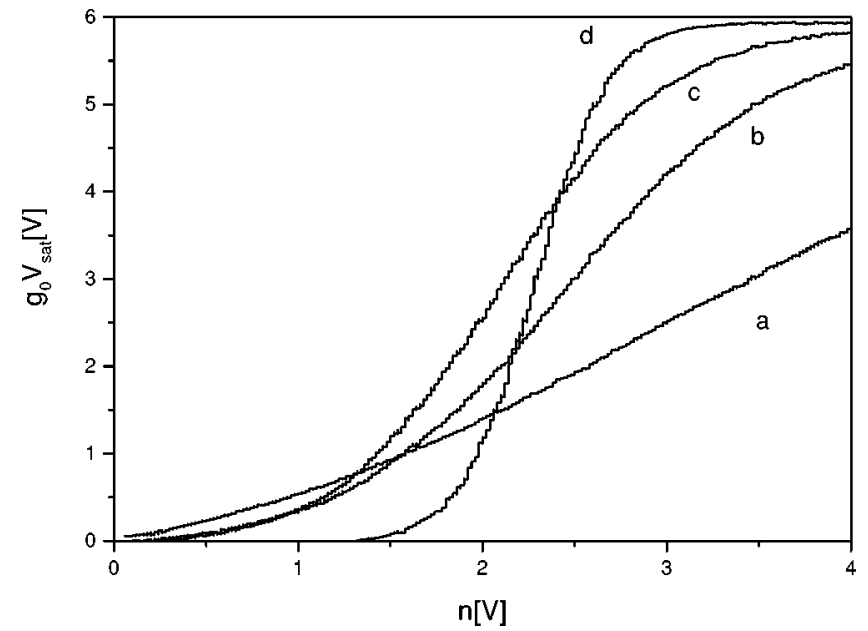

FIG. 6. $g_{0} v_{\text {sat }}$ as a function of $n$ : (a) $\lambda=0.7 \mathrm{~V}^{-1}, \lambda n_{0}=3$, (b) $\lambda=1.7 \mathrm{~V}^{-1}, \lambda n_{0}=4, \quad$ (c) $\lambda=3 \mathrm{~V}^{-1}, \lambda n_{0}=6$, and (d) $\lambda$ $=10 \mathrm{~V}^{-1}, \lambda n_{0}=20$.

pulses. For the highest value of $\lambda$, we get to miss the first pulse and perceive the saturating nature of the nonlinearity in the amplitude of the responses in Fig. 7.

\section{BIOLOGICAL EXPERIMENTS}

The experiments were performed in the nervous system of the leech. The body architecture of this annelid is fairly simple, and this simplicity is reflected in the structure of its nervous system. There are 21 ganglia innervating each midbody segment; the anterior four ganglia and the posterior seven ganglia are fused to form a head and a tail brain. Each ganglion contains $\approx 200$ bilateral pairs of neurons, as well as a few unpaired neurons [17]. The neuronal cell bodies form a single layer of outer cortical cells that are distributed among six packets on the dorsal and ventral surfaces. The neurons are monopolar, with their processes projecting into the central neuropil, where they make synaptic contacts. In each segment, neurons innervate the periphery via two laterally

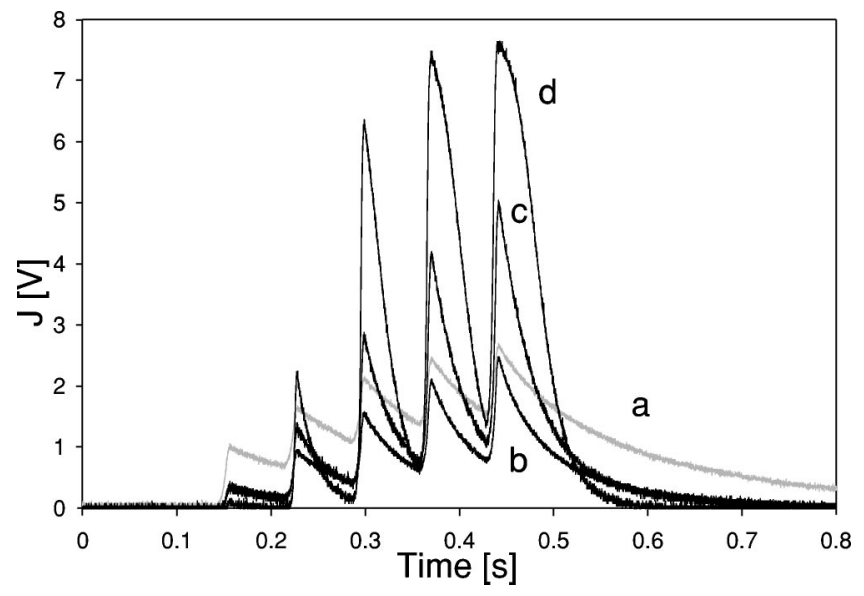

FIG. 7. Evolution of $J$ when we forced the excitable circuit with a train of five pulses of $7 \mathrm{~ms}$ separated by $66 \mathrm{~ms}$ : (a) gray line, linear regime of the sigmoidal function; (b) $\lambda=0.7 \mathrm{~V}^{-1}, \lambda n_{0}=3$; (c) $\lambda=1.5 \mathrm{~V}^{-1}, \lambda n_{0}=3$; and (d) $\lambda=3 \mathrm{~V}^{-1}, \lambda n_{0}=8$. 

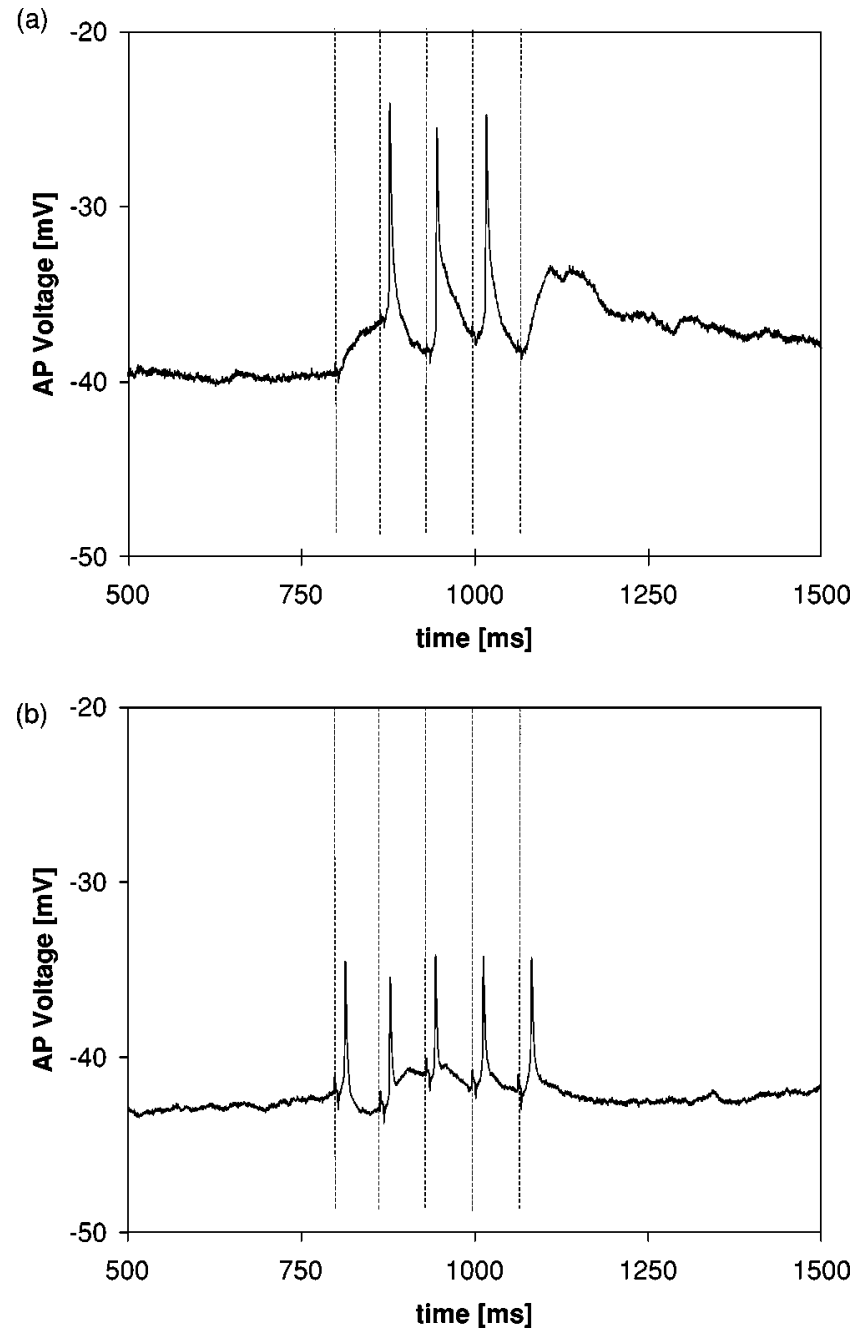

FIG. 8. Voltage at an AP neuron when a P neuron was excited with five pulses for two different ganglia (a) and (b). In both figures, we have similar deporarizations of the cell. The dashed vertical lines show the times where the pulses used to excite were applied.

directed pairs of nerves. The 21 midbody ganglia are very similar to each other; a standard map identifies neurons by somatic location, size, and physiological properties [18].

Many of the neurons within a leech ganglion have been characterized in terms of their function and their physiological properties. Moreover, the circuits underlying some of the major motor behaviors have been described in detail.

In the present work, we have used a well-characterized synaptic interaction between the mechanosensory, sensitive to pressure, (P) cell and the anteropagoda (because of the action potential shape) (AP) neuron for an initial evaluation of the electrical device developed. When a P neuron is stimulated with a train of action potentials, the potential across the AP membrane is depolarized. This is mostly induced by a direct P-AP interaction [19].

The experiments were performed dissecting out a midbody ganglion that was pinned to Sylgard, ventral side up, in a chamber with a saline solution (concentration in $\mathrm{mM}$ : $\mathrm{NaCl}, 115 ; \mathrm{KCl}, 4 ; \mathrm{CaCl}_{2}, 1.8 ; \mathrm{MgCl}_{2}, 1$; trismaleate, 4.6; (a)

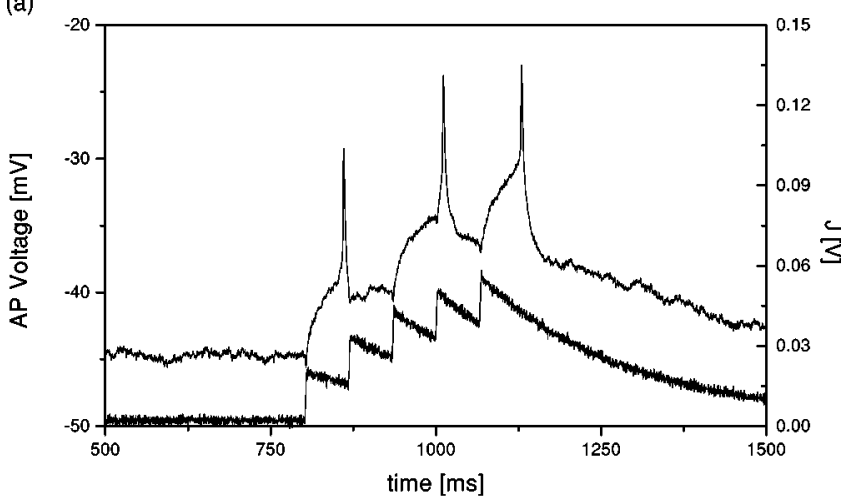

(b)

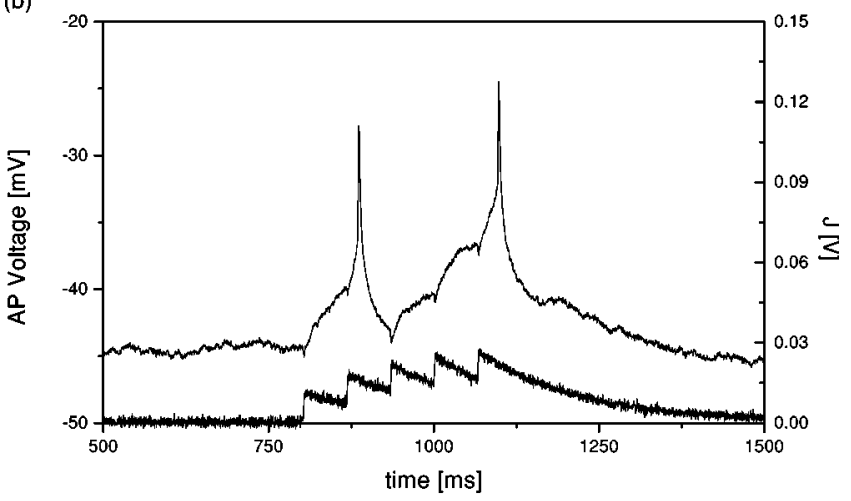

(c)

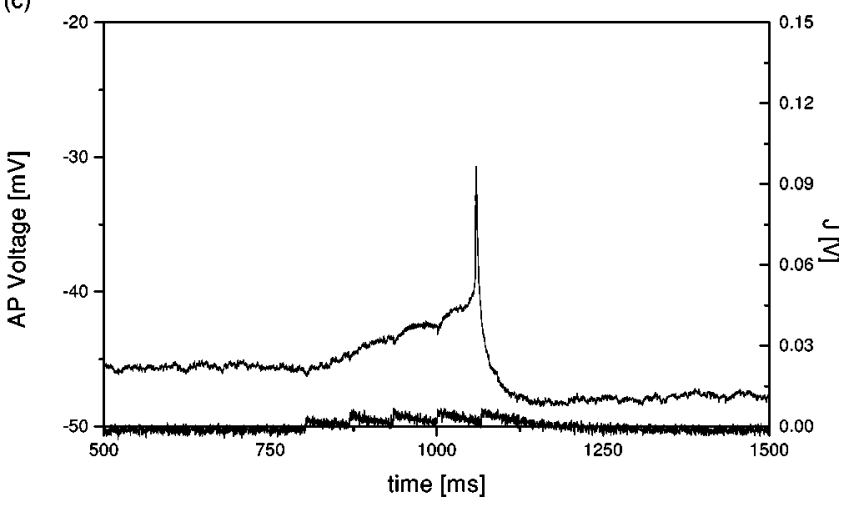

FIG. 9. Response of the AP neuron in the mixed electronicbiological experiences for different values of the neurotransmitter loss rate $\gamma$ [see Eq. (4)]: (a) $\gamma=37 \mathrm{~s}^{-1}$, (b) $\gamma=55 \mathrm{~s}^{-1}$, and (c) $\gamma$ $=100 \mathrm{~s}^{-1}$. The injected current $J$ is shown in the lower trace.

trisbase, 5.4; and glucose, $10 ; p \mathrm{H} \mathrm{7.4).} \mathrm{The} \mathrm{neurons} \mathrm{were}$ stimulated and recorded through intracellular electrodes connected to an amplifier (Axoclamp 2B, Axon Instruments, Foster City, California) operating in the current-clamp configuration. The electrodes were pulled from borosilicate capillary tubing filled with a potassium acetate solution $(3 \mathrm{~mol} /$ 1), and were bridge balanced. The recordings were digitized using a Digidata 1320 interface and acquired using Clampex protocols (pClamp 8.0.2, Axon Instruments) at sampling frequencies of $3 \mathrm{kHz}$. The $\mathrm{P}$ cells or the analog device were stimulated by trains of suprathreshold step pulses $(2-4 \mathrm{nA}, 7$ ms) separated by $66 \mathrm{~ms}$ delivered by a stimulator Master 8 (AMPI, Jerusalem), which was triggered by the acquisition software. 


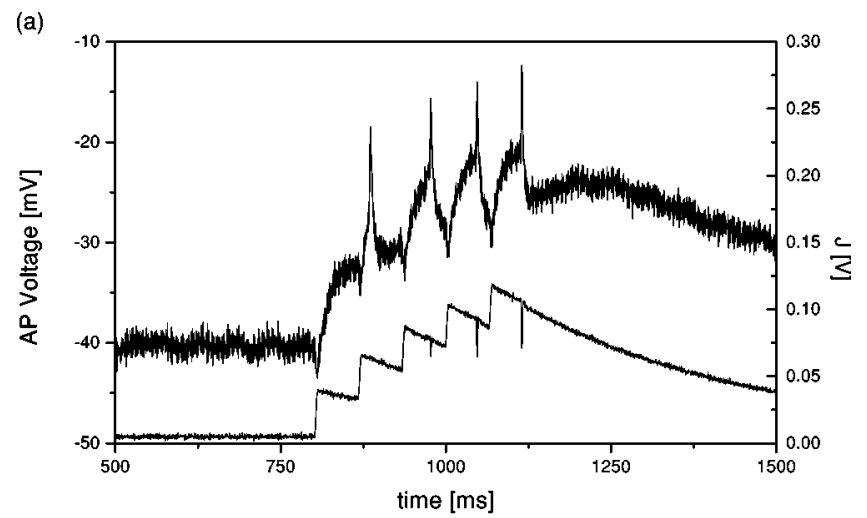

(b)

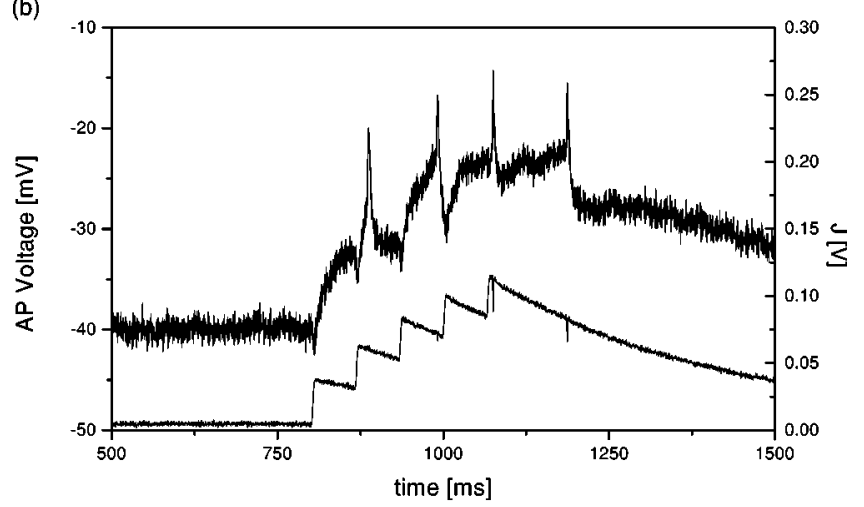

(c)

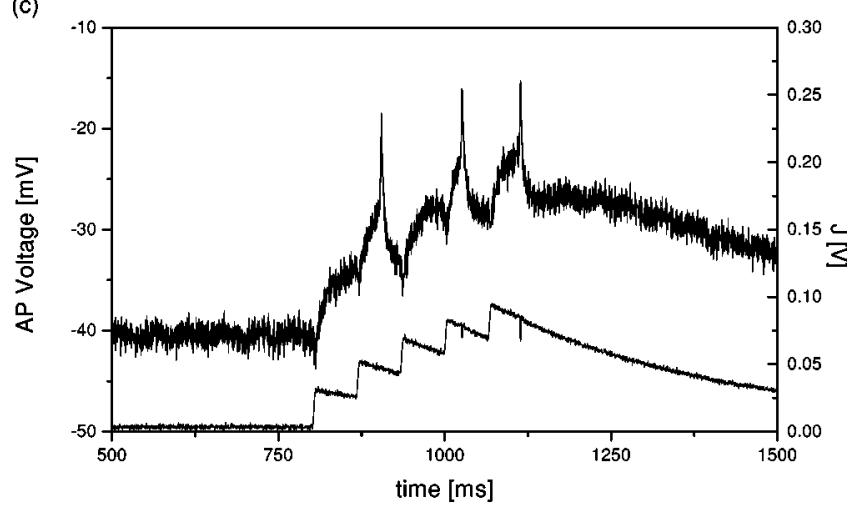

(d)

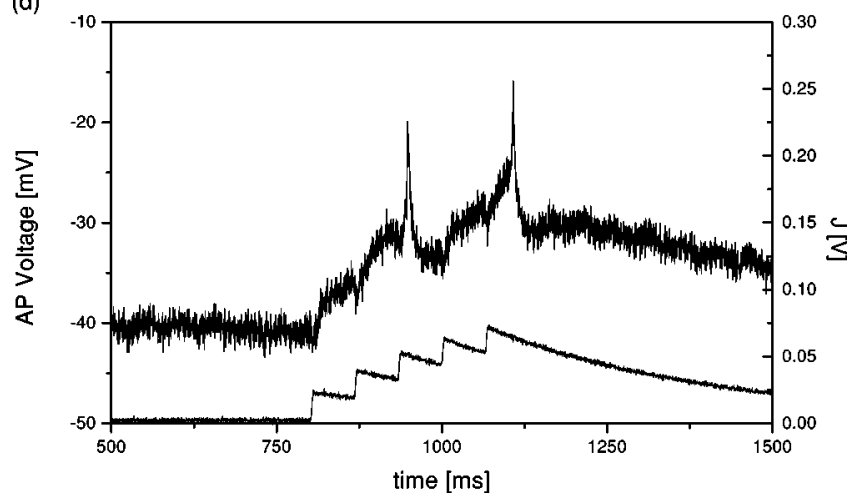

FIG. 10. Response of the AP neuron in the mixed electronicbiological experiences for different values of the amplification parameter: (a) $R_{12}=5.5 \mathrm{k} \Omega$, (b) $R_{12}=4.7 \mathrm{k} \Omega$, (c) $R_{12}=3.8 \mathrm{k} \Omega$, and (d) $R_{12}=3.0 \mathrm{k} \Omega$. The injected current $J$ is shown in the lower trace.

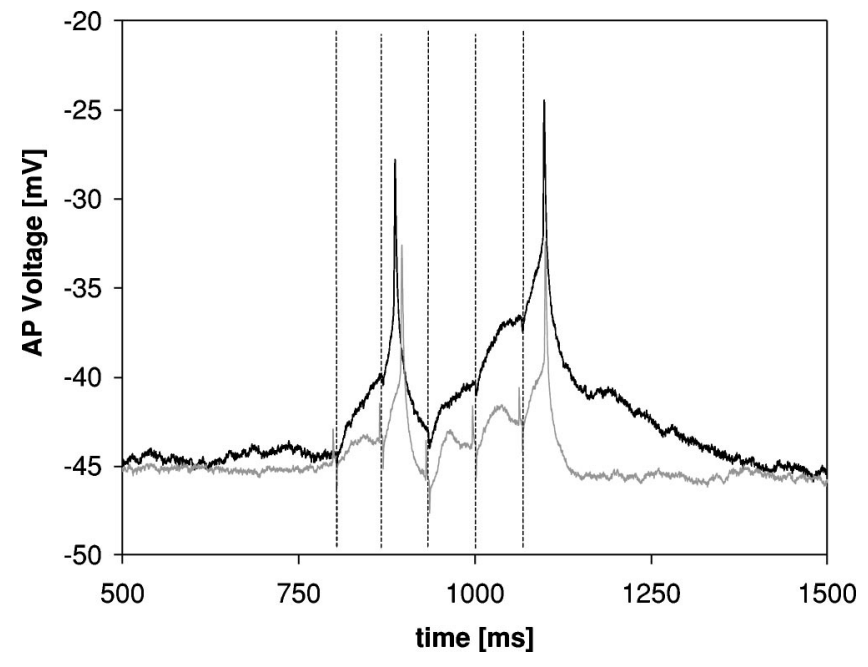

FIG. 11. Response of the AP neuron for mixed electronicbiological (dark line) and a biological (gray line) experiences.

In each experiment $(n=10)$, we first recorded the electrophysiological responses of an AP cell to a P cell stimulation, and then we tested the responses to the electronic device. The latter was connected to the AP neuron in the following way. In the point denoted by $V_{v, \text { pos }}$ in Fig. 2(c), we sensed the membrane potential of the AP neuron. In most experiments, $V_{\text {rev }}$ was fixed at zero, while the output $J$ [see Fig. 2(c)] was injected into the AP neuron through the input connection of the amplifier recording the AP neuron. The aim of these series of experiments was to find out whether the fitting of the parameters in our device could lead to results similar to those obtained by stimulating the $\mathrm{P}$ cell.

Let us now discuss the biological experiences. Figure 8 shows typical responses of AP neurons that display depolarizing voltage deflections, time locked to the $\mathrm{P}$ action potentials, that occasionally reached the threshold. On the other hand, Figs. 9 and 10 show some recordings of an AP neuron obtained by the stimulation of the electronic cell whose output was connected to the AP electrode. The traces in Fig. 9 were obtained by varying the neurotransmitter loss rate $\gamma$ [see Eq. (4)]. The kinetics of the actual injected current is shown below each AP recording. The traces in Fig. 10 were obtained by varying the conductivity $g_{0}$. The larger is the value of $g_{0}$, the larger is the amplitude of the AP responses and, therefore, the most likely it was to evoke an action potential.

Variation of $\gamma$ and $g_{0}$ allowed an adjustment of the electronic device output. Figure 11 compares the responses of the same AP neuron to P cell stimulation and to the stimulation of the electronic cell under the following configuration: $\gamma$ $=55 \mathrm{~s}^{-1}, g_{0}=5.5 \mathrm{k} \Omega$. This corresponds to an experiment in which the synaptic parameters were adjusted in order to achieve responses in the AP neuron displaying the same number of spikes and at the same timing. Although whether (and in which cases) the precise timing is the pertinent element that ultimately participates in the process of conveying information in nervous systems, is still an active area of research [20], the possibility of fitting these times with our artificial synapse suggests that the degree of simplification 
implied in the model leaves room for obtaining realistic results.

\section{CONCLUSIONS}

In this work, we report the construction of an electronic device that is ruled by a set of equations modeling the excitable nature of neurons and many of the properties of neural synapses. The excitable system built behaves as an excitable system of class I (which under parameter changes loses its excitability through an Andronov bifurcation). The type of excitability displayed by a neuron is relevant at the moment of exploring the dynamics of large sets. In this work, we only took the first steps in this direction, by connecting one of these devices to a biological neuron. Since in this preparation many neurons display this kind of excitable behavior, this electronic device will allow us to build more complex circuits.

In terms of reproducing the number and timing of spikes in a postsynaptic neuron, our device was successful. Therefore, it is possible to conclude that the degree of approximation implied in the models whose equations rule our device are satisfactory (at the scale of description in which the num- ber and the timing of spikes are relevant). At the same time, the validation or refutation of the simplifications involved in the description of the neural system is not the numerical output of a model built ad hoc, but the real response of the biological system under study. For this reason, this mixed technology is an optimal laboratory to study the scope and limitations of mathematical models.

The preparation used to build our mixed circuits is a challenging one. A leech displays a reasonably bounded number of simple (yet nontrivial) tasks which will allow us to explore, in further works, the architecture of more complex circuits. In this sense, this preparation is the most interesting candidate to explore the consequences of changes in connectivity structures. For example, some complete circuits involved in the processing of pressure sensory inputs and output motor commands have been identified [21,22]. In future works, we expect to address the construction of mixed circuits like this one.

\section{ACKNOWLEDGMENTS}

This work was partially funded by the UBA, the CONICET, and the Fundacion Antorchas.
[1] A. Szucs, P. Varona, A.R. Volkovskii, H.D.I. Abarbanel, M.I. Rabinovich, and A.I. Selverston, J. Comput. Neurosci. II, 1 (2000); Y. Yarom, Neuroscience 44, 263 (1991); S. Le Masson, A. Laflaquiere, T. Bal, and G. Le Masson, IEEE Trans. Biomed. Eng. 46, 638 (1999); M.F. Simoni, G.S. Cymbalyuk, M.Q. Sorensen, R.L. Calabrese, and S.P. DeWeerth, in Advances in Neural Information Processing Systems 13, Neural Information Processing Systems (NIPS) 2000, Denver, edited by Todd K. Leen, Thomas G. Dietterich, and Volker Tresp (MIT Press, Cambridge, MA, 2001), p. 173; R. Jung, E.J. Brauer, and J.J. Abbas, IEEE Trans. Neural Syst. Rehabil. Eng. 9, 319 (2001).

[2] M. Feingold, D.L. Gonzalez, O. Piro, and H. Viturro, Phys. Rev. A 37, 4060 (1988).

[3] A.L. Hodgkin and A.F. Huxley, J. Physiol. (London) 117, 500 (1952).

[4] R. FitzHugh, Biophys. J. 1, 445 (1961).

[5] J.S. Nagumo, S. Arimoto, and S. Yoshizawa, Proc. IRE 50, 2061 (1962).

[6] J.D. Murray, Mathematical Biology (Springer, Berlin, 1989).

[7] F. Moss, J.K. Douglass, L. Wilkens, D. Pearson, and E. Pantazelou, Ann. N.Y. Acad. Sci. 706, 26 (1993).

[8] J.L. Hindmarsh and R.M. Rose, Proc. R. Soc. London, Ser. B 221, 87 (1984).
[9] F. Hoppensteadt and E.M. Izhikevich, Weakly Connected Neural Networks (Springer, New York, 1997).

[10] A.C. Ventura, G.B. Mindlin, and S. Ponce Dawson, Phys. Rev. E 65, 046231 (2002).

[11] J.E. Spiro, M.B. Dalva, and R. Mooney, J. Neurophysiol. 81, 3007 (1999).

[12] C. Koch, Biophysics of Computation (Oxford University Press, Oxford, 1999).

[13] B. Hu and C. Zhou, Phys. Rev. E 63, 026201 (2001).

[14] A.M. Yacomotti, M.C. Eguia, J. Aliaga, O.E. Martinez, G.B. Mindlin, and A. Lipsich, Phys. Rev. Lett. 83, 292 (1999).

[15] M.C. Eguia, M.I. Rabinovich, and H.D.I. Abarbanel, Phys. Rev. E 62, 7111 (2000).

[16] J.M. Mendez, R. Laje, M. Giudici, J. Aliaga, and G.B. Mindlin, Phys. Rev. E 63, 066218 (2001).

[17] E.R. Macagno, J. Comp. Neurol. 190, 283 (1980).

[18] R.E. Coggeshall and D.W. Fawcett, J. Neurophysiol. 27, 229 (1964).

[19] K.J. Muller, J.G. Nicholls, and G.S. Stent, Neurobiology of the Leech (Cold Spring Harbor, New York, 1981).

[20] F. Rieke, D. Warland, R. de Ruyter van Steveninch, and W. Bialek, Spikes: Exploring The Neural Code (MIT Press, Cambridge, MA, 1997).

[21] J.E. Lewis and W.B. Kristan, Nature (London) 391, 76 (1998).

[22] S.R. Lockery and W.B. Kristan, J. Neurosci. 10, 1816 (1990). 